\title{
CORVETTE: A COOPERATIVE WORKFLOW DEVELOPMENT EXPERIMENT
}

\author{
K. Baïna ${ }^{2}$, F. Charoy ${ }^{2}$, C. Godart ${ }^{2}$, D. Grigori ${ }^{2}$, S. el Hadri ${ }^{2}$, H. Skaf ${ }^{2}$, \\ S. Akifuji ${ }^{1}$, T. Sakaguchi ${ }^{1}$, Y. Seki ${ }^{1}$, M. Yoshioka ${ }^{1}$ \\ ${ }^{\prime}$ Hitachi SDL, Kawasaki Lab., 1099, Ohzenji, Asao-ku, Kawasaki-shi, Kanagawa-ken, 215- \\ 0013 JAPAN, http://www.sdl.hitachi.co.jp, \\ ${ }^{2}$ ECOO Team, LORIAIINRIA, Campus Scientifique, BP 239, 54506 Vandoeuvre Cedex, \\ FRANCE, http://www.loria.fr/equipes/ecool, \\ Karim.Baina@loria.fr \\ Workflow systems are now successfully used in numerous administrative and \\ production applications. Following this success, a lot of research has been \\ launched to keep workflow technology supporting a larger range of \\ applications. This paper reports on CORVETTE project, an experience in \\ developing a cooperative workflow system by integrating a commercial \\ workflow system developed by Hitachi Lid. with a cooperation technology \\ developed by INRIA. By cooperative workflow system, we mean a workflow \\ system that has the capability to manage cooperative behaviours \\ characteristic of creative applications, typically co-design and co-engineering \\ processes, where interactions between activities are more subtle than in \\ traditional applications. More precisely, Corvette was targeted to support \\ coordination processes of a virtual team working over Internet.
}

\section{INTRODUCTION}

Workflow systems are now widely and successfully used in a large number of administrative and production applications. To address a larger range of applications, a lot of research has been launched to surpass the limits of current workflow technology (Suchmann 1987; Reuter 1995; Agostini and De Michelis 1996; Nutt 1996; Weske 1996; Ellis and Maltzahn 1997; Dourish 1998; Han, Sheth et al. 1998; Reichert and Dadam 1998; Casati 1999; Georgakopoulos 1999; Heinl, Horn et al. 1999; Grigori, Charoy et al. 2001). Among them, some researches (Georgakopoulos 1999; Joeris 1999; Grigori, Charoy et al. 2001) concentrate on supporting creative processes, typically co-design and co-engineering processes, where interactions between activities are more subtle than in traditional applications. We call a cooperative workflow system, a workflow system that has the capability to manage cooperative behaviours characteristic of creative applications. This paper reports on CORVETTE ${ }^{\mathrm{ii}}$ project, an experiment in developing a cooperative workflow system by integrating a commercial workflow system developed by Hitachi Ltd. with a cooperation technology developed by INRIA. More precisely, Corvette was targeted to support coordination processes of a virtual team working 
over Internet. It is organized as follows. Section 2 motivates cooperative workflow. Section 3 describes CORVETTE system design. Section 4 sketches the implementation. Section 5 synthesizes, finally, the experience and discusses some learned lessons.

\section{COOPERATIVE WORKFLOW - MOTIVATIONS}

Current workflow models are mainly concerned with the automation of administrative and production processes. These processes coordinate well defined activities which execute in isolation, i.e. synchronize only at their start/terminate states. If these models apply efficiently for a class of applications, they show their limits when one wants to model the subtlety of cooperative interactions as they occur in more creative processes, typically co-design and co-engineering processes. Cooperative workflow has been mainly introduced to surpass these limits :

Definition1: "a cooperative workflow is a workflow where some activities executing in parallel can share some intermediate results during their execution" (Godart 1993).

Cooperative workflow has the capability to synchronize activities at any point of their executions and thus insures the coordination between designers (Grégori 1999).

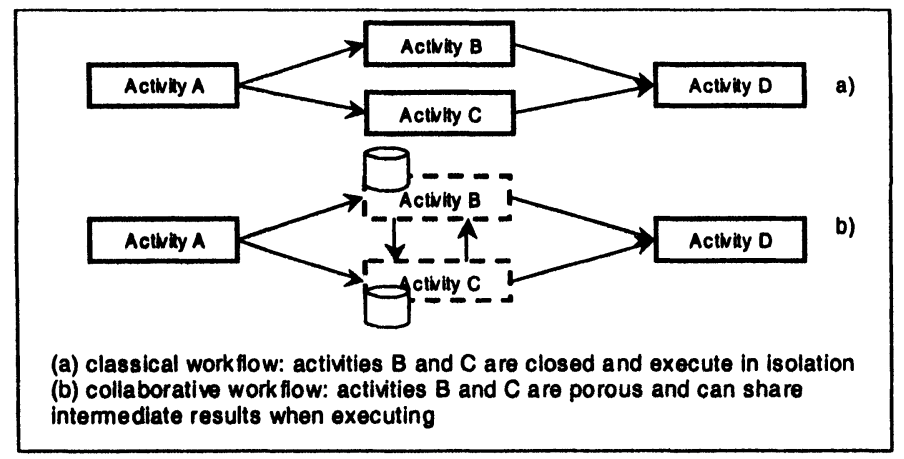

Figure 1 - From classical to cooperative workflow

To illustrate that, let us consider a building trade process in which three partners (an architect, a research engineer and a building contractor) cooperate. The architect has the responsibility for producing plans responding to a set of requirements given by a client. Based on plans of the architect and on its proper expertise, the research engineer makes main technological choices. The building contractor has the responsibility for manufacturing wood components and finally for assembling them on building site. This process is roughly described in Figure 2(a). A traditional, i.e. sequential, execution of this process is depicted Figure 2(b). But real processes do not execute in this way. Processes corresponding to the three partners are more intricate and execute rather in parallel than in isolation: they exchange documents when executing with the objective to have a rapid feedback and to point out risks in 
the construction as soon as possible. In general, definition of a model as Figure 2(b) can delay the evidence a design problem and contribute to a poor acceptance of the workflow system on working sites. A better way to do things is to allow the architect, the engineer and the contractor to cooperate by early exchanging intermediate results (e.g. draft documents) when operating. That is illustrated in Figure 2(c) beside the fact that this organization can dramatically decrease the duration of a process.

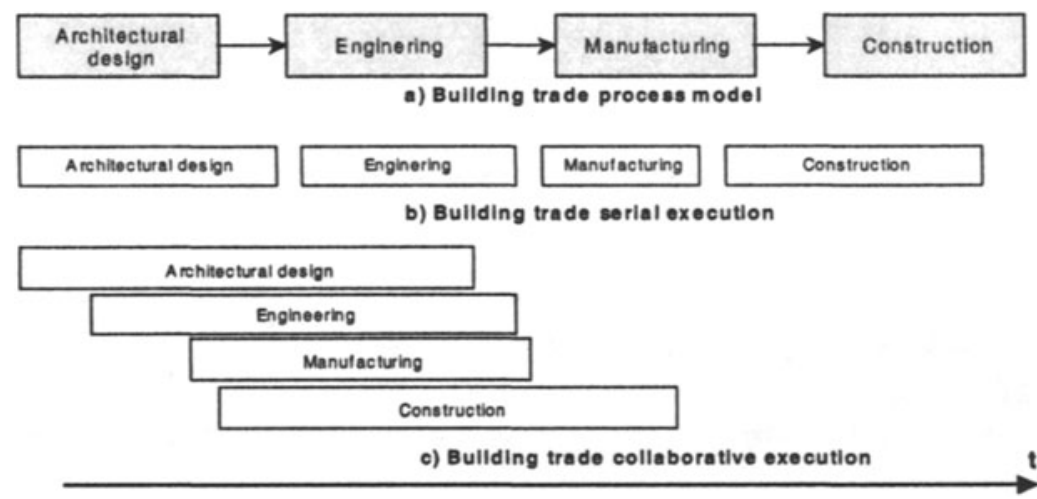

Figure 2 - Building trade example

Enabling interactions between parallel activities is a typical cooperation characteristic within creative applications we are concerned with. One important question at this point is : can a traditional workflow system model and enact efficiently such interactions ? In more admitted definitions of a workflow management system (WfMC; Jablonski and Bussler 1996; Leymann and Roller 1999), an activity is a black box with an input container fulfilled before the activity starts its execution and an output container fulfilled when the activity ends, thus forbidding visibility of intermediate results.

Obviously, such a WFMS cannot easily support such interactions. One can try to model it with iterations, but the limits of this solution is easily reached. Interactions are difficult to forecast and their number increases exponentially with the number of activities (Godart 1993). Moreover, for practical opportunity, some products allow activities to interact when executing, but in this case, semantics of parallel executions become quite unclear, or rather is delegated to the applications, under the responsibility of programmer. As far as current systems cannot easily model cooperative behaviours there is, really, a need for cooperative workflow technology.

\section{CORVETTE DESIGN}

The objective of CORVETTE project is to experiment the development of a cooperative workflow system to coordinate a virtual team cooperating through a network, typically Internet. Cooperation has to be taken there in the sense of the ECOO cooperation model that we will present below. The system must be implemented by "connecting" Hitachi WorkCoordinator (WCO 2001) workflow 
management system and ECOO Motu system (Motu 2001), which provides complementary cooperation services, and especially $\mathrm{COO}^{1}$ cooperative transactions. This implementation must not touch WorkCoordinator source code. In other terms the only means of communication with WorkCoordinator are its CORBA IDL API and indirectly its related Oracle database.

This section is organized in three steps. First, we introduce the contributing technologies, second, we present the important design decisions, and third, we illustrate the detailed architecture of CORVETTE.

\subsection{Contributing technologies}

We start with the ECOO cooperation technology represented by Motu system, then we introduce Hitachi workflow technology within WorkCoordinator system.

\subsubsection{ECOO Cooperation technology - Motu System}

ECOO cooperation model is based on the ability for an activity to publish an intermediate result, as introduced in section 2. More precisely the ECOO cooperation model is characterized by four generic cooperation design patterns as depicted below :

- Producer/consumer. Two activities follow a producer/consumer pattern if one has the responsibility to create/modify an object and the other reads this object to integrate it in its own work. Producers and consumers can momentarily see different versions of the same object, but the consumer must retrieve the final producer version.

- Redactor/reviewer. Two activities follow a redactor/reviewer pattern if one (the redactor) create/modify an object and the second (the reviewer) reads one or several successive versions of this object with the objective to review it. The corresponding review objects are also shared in their turn.

- Cooperative write. Two activities follow a cooperative write pattern if they develop a common object, work on two different versions and merge frequently their modifications. They have, at the end, to agree on a common final version.

- Concurrency. Two activities follow a concurrency pattern if they must execute in isolation. In cooperative processes, if same activities must be allowed to share some results, some must be allowed to execute in isolation with respect to others. Based on ECOO cooperation model, Motu system (Motu 2001) is a tool in the vein of BSCW (BSCW), TeamScope (Teamscope) or (Sourceforge). It proposes, among other functionalities, workspace management, communication, coordination, group awareness (CHI 1997) etc. The main Motu functionalities used in Corvette are the workspace manager and the $\mathrm{COO}$-transaction manager that have been streamlined to manage consistency of non linear versioned document exchanges.

The COO-transaction protocol asserts concurrency atomicity of cooperative

${ }^{1} \mathrm{COO}$ stands for COOperation transaction model 
processes, i.e. correctness of interactions between processes exchanging results when executing. For this purpose, each process is encapsulated in a COO-transaction. Each COO-transaction executes in its private workspace and COO-transactions cooperate by transferring intermediate results between their workspaces. The COO-transaction protocol is a set of rules which constrains these transfers. They are intuitively depicted in the following (for a more formal definition see (Canals, Godart et al. 1998)) :

- a result produced before the end of a transaction is always an intermediate result of this transaction. Users can produce an intermediate result (operation $I R$-write),

- we call final result, a result produced at the end of a transaction. All final results are produced atomically during the execution of the terminate operation of the transaction. A transaction that produces an intermediate result must produce a corresponding final result. The protocol collects all the objects that were $I R$ written by the activity and automatically produces a final result for each of them during the termination phase of the activity,

- if a transaction has read an intermediate result, then it must read the corresponding final result. The system maintains a dependency relationship between activities to memorize the fact that a transaction reads an intermediate result of another one. When a transaction $A_{l}$ reads the intermediate value of an object $x$ produced by a transaction $A_{0}$, a dependency $A_{l} \rightarrow A_{0}$ is created. When the transaction $A_{I}$ reads a value of $x$ and $A_{0}$ is terminated (i.e. when it has produced its final result), the dependency is removed,

- a transaction cannot terminate if it is still dependent on another one. If a transaction tries to terminate without reading the final value of some object after a previous access to an intermediate value of this object, the terminate operation is aborted and the transaction remains active,

- transactions involved in a cyclic dependency graph form a group of transactions,

- a group-member transaction can start a group termination by trying to terminate itself. The terminate operation in this case produces a set of potentially final results and changes the state of the transaction from active to ready to commit (RTC),

- when a group-member transaction tries to terminate and all the other groupmembers are in the RTC state, then all the transactions are terminated simultaneously. Potentially final results are definitely promoted to final results,

- when a group-member transaction tries to terminate and another group-member is still active, then the former produces new potentially final results and enters RTC state,

- if a group member produces a new intermediate result during the group termination phase, then this termination tentative is aborted, and all the groupmembers re-enter the active state. This is the way for a transaction to clearly indicate its disagreement with the object values produced by the group, and to ask for more work on the shared object.

For more about COO-transactions, see (Canals, Godart et al. 1998) and (Skaf, Charoy et al. 1999) (for the relationship between concurrency, atomicity and consistency). 


\subsubsection{Hitachi Workflow technology - WorkCoordinator system}

WorkCoordinator (WCO) considers a workflow as a control flow graph with activities as nodes and inter-activities transitions as edges (as defined by the $\mathrm{W} f \mathrm{MC}^{2}$, i.e. and-join, and-split, or-join, or-split, sequence, etc...). An activity has a description (text), a deadline (in day), and a post condition (SQL statement) which defines when the activity can complete. An activity is associated to a non empty set of work items. A work item is an atomic job that symbolises the place where real work is done. Contrary to activities, there is no control flow scheduling work item execution. A work item has a description (text), a casting rule (SQL statement) that identifies the work item performer, a pre-condition and a post-condition (SQL statement) which define respectively when it can start and complete. The parallel execution of activities or work items enables data to be shared when executing (objects, rows and files) and provides flexibility and co-operation. However, parallel execution consistency control concerning data access is left on the responsibility of programmers. WCO (WorkCoordinator) workflow model emphasises control flow but does not describe data flow between neither activities nor work items. WCO separates the process definition model (including process control flow definition) and the process execution context (including casting rules definitions, used application information, condition definitions and rule definitions, etc.). While the former is encapsulated in WCO framework, the latter is handled by extern database management systems (Hitachi or Oracle RDBMS). Thanks to this flexibility of WCO architecture, the modification of a process execution context is possible without process definition model alteration. Moreover, WCO keeps possible to modify process definitions at runtime. Thus, cohabitation of several versions of the same processes can be insured. Beside these capabilities, of definition and enacting processes, $W C O$ offers facilities for monitoring the life cycle of process instances, activities and work items. Finally, it integrates a CORBA API which is WfMC Interface 2 compliant, thus allowing external systems to invoke some of its methods. For this purpose, it uses Visigenic broker for $\mathrm{C}++$.

\subsection{Design choices}

Design choices were seriously directed by the constraint of not modifying the WorkCoordinator system. This accelerated our choice for cooperative process modelling, simplified discussions about architecture, but limited the cooperation model finally implemented in Corvette. However, note that, on the first hand, clearly distinguishing between what is matter of workflow from what is matter of data flow and concurrency is a good policy in matter of software architecture, and on the other hand, the objective of Corvette is to make experiments, not to develop directly a product.

${ }^{2}$ WfMC : Workflow Management Coalition www.wfmc.org 
Corvette cooperative process modelling. The question is how to model cooperative processes ? In the ECOO project, we study two complementing approaches. The first approach consists in providing new workflow operators to explicitly point out where cooperation is possible (Godart 1993) these "cooperative operators" extend the set of traditional operators ${ }^{3}$. The second consists in modelling a cooperative process in the same way than traditional production processes, using the same set of operators, but interpreted in another, i.e. cooperative, way. To simplify, in a traditional interpretation, activities are seen as black boxes, while in a cooperative interpretation they are seen as white boxes (being allowed to share intermediate results).

In Corvette, we chose the second alternative which has two qualities :

- first, it corresponds to (a) reality : in process books, administration processes are described in the same way than design processes; it is the reader interpretation that changes depending on its know-how of application domain (idea depicted in Figure 2)

- second, introducing cooperation has no impact on process modelling and allows to reuse the $W C O$ process modeller, to model Corvette cooperative processes, as it is, thus fulfilling the constraint of not modifying WCO source code (but of course transferring the problem to process enactment).

Another decision was, without losing in generality, to have one work item per activity.

Corvette Cooperation Model. Based on the decision to not to modify neither WCO model nor WCO source code, an arising question is : which cooperation, or which cooperation patterns, is it still possible to model with this constraint ? In fact, and fortunately, WCO is mainly concerned with control flow and is very permissive regarding data flow. In fact, it imposes no constraint on data flows and cooperation between two work items, and in our context between two parallel activities, is allowed. However, an important restriction came from the fact that, as a WCO activity can execute only when its preceding activities have terminated their executions, only activities in parallel branches can cooperate. This restriction has an impact on process modelling. We think that Producer/consumer and Redactor/reviewer refer by nature succeeding activities. In other terms, either these patterns are not allowed, or their implementation implies some contra-nature modelling.

\subsection{Corvette architecture}

Corvette Software Components. WorkCoordinator system handles the process modelling and the enactment and coordination of workflow activities letting data management on the programmer responsibility. Motu System supports Cootransactions with managing versioned document workspace for each coo-transaction. The CORVETTE architecture had, thus, integrated tow main component, the first handling workflow activities (control flow) and the second handling Cootransactions and versioning (data flow). In this architecture, it was not possible to modify WorkCoordinator, so the decision was quickly oriented towards the

${ }^{3}$ Typically and-join, and-split, or-join, or-split, sequence as defined by WfMC. 
definition of a mediator between WorkCoordinator, Motu and users without intervening in contributing components source code. But this was not the single justification of this architecture: we think that clearly distinguishing between a component for control flow management and another for dataflow management is a good software architecture in middleware programming context.

Figure 3 depicts the overall Corvette architecture. A Corvette Client component assumes the mediator role between one user, WorkCoordinator and Motu transactions and workspaces server. In other terms, there is one Corvette Client per user, but a user can simultaneously be performer of several workitems, executing in different workspaces. Concurrency between workitems interfaced by one or several Corvette clients is handled by the Motu transaction manager. See (CorvetteManual 2001) for more about CORVETTE interface.

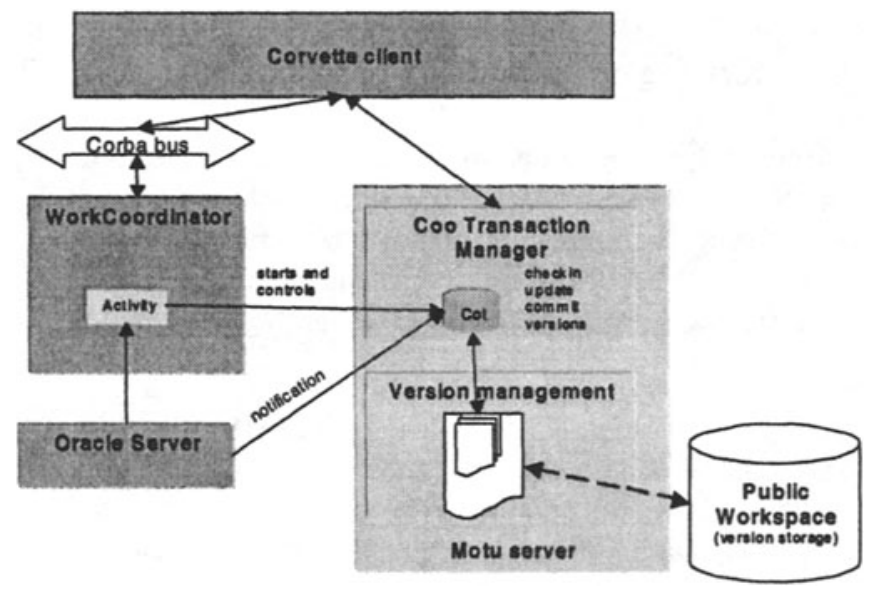

Figure 3 - Corvette general design

Corvette plugging rules. The main issue in defining CORVETTE client was the ability to establish a correspondence between WorkCoordinator process definition concepts and the $\mathrm{COO}$ transaction concept, or how to encapsulate a WorkCoordinator unit of work in $\mathrm{COO}$ transaction?

\begin{tabular}{|l|l|}
\hline \multicolumn{1}{|c|}{ Work Item state } & Coo Transaction state \\
\hline Initial & Initial \\
\hline Performing & Executing \\
\hline & Waiting Commit \\
\hline & RTC \\
\hline Completed & Terminated \\
\hline
\end{tabular}

Figure 4 - WorkCoordinator work item / COO transaction states Mapping

As, in WorkCoordinator, real work is executed in work items, the decision was to associate each work item to a $\mathrm{COO}$ transaction. As work items can run in parallel, cooperation in the sense of intermediate result sharing can occur. And encapsulating work items in $\mathrm{COO}$ transaction asserts consistency of cooperation in the sense of the $\mathrm{COO}$ protocol. As work items are started automatically by the Work Coordinator 
execution engine as soon as their activation conditions are fulfilled (e.g. the preceding work item has completed), this must be detected in order to create the corresponding $\mathrm{COO}$ transaction. Reciprocally, termination of a work item must be done in coordination with its corresponding $\mathrm{COO}$ Transaction. This means that termination condition of the work item and of the $\mathrm{COO}$ transaction must be fulfilled at the same time. An other issue concerns termination of a group of $\mathrm{COO}$ transactions. When several COO transactions are grouped due to cyclic dependencies between them, they must terminate simultaneously, following a kind of two phases termination protocol. That means that all the corresponding work items must also terminate simultaneously. To implement this capability, one new state was introduced (WaitingCommit) in the COO-transaction model. Figure 4 depicts this mapping.

The role of CORVETTE client is to manage this mapping. This is mainly performed in the create work item, perform work item, open workspace and terminate work item Corvette commands :

- corvette create work item : this command overwrites the WorkCoodinator create work item command to create, for the created work item, the associated transaction structures, including the corresponding private workspace,

- corvette perform work item : this command overwrites the WorkCoordinator perform work item command to manage the associated transaction (pushing it in executing state),

- corvette open workspace : this command allows to create and populate the private workspace associated to the transaction with necessary work item enactment artefacts,

- corvette terminate work item : this command overwrites the WorkCoordinator terminate work item command to manage the associated transactions. It is easy to understand the central role of this complex command. Let us explain its algorithm :

1. if the corresponding transaction is not in a group of transactions :

1.1.1. if it is dependent on another one (has read an intermediate result of another active transaction), it must wait for this other transaction to terminate,

1.1.2. if not, it enters the waiting commit state and asks the encapsulated work item to terminate,

1.1.2.1. if the work item terminates (all its termination conditions are fulfilled), the transaction commits,

1.1.2.2. if not, the transaction returns to the executing state,

2. if the corresponding transaction is member of a transaction group :

2.1.1. if there are still transaction(s) of the group executing, the transaction enters the ready to commit (RTC) state,

2.1.2. if all other transactions are in RTC state, the transaction triggers the termination of all the work items of the group and enters the waiting commit state,

2.1.2.1. if all work items terminate, all transactions of the group are committed,

2.1.2.2. if not, as a work item cannot go back to the performing state, a manual recovery phase is launched.

Problems with a "work item group" termination: due to cycles in cooperation, a 
group of work items, associated to a group of transactions, have to terminate simultaneously. Unfortunately, a problem occurs when a sub-set of such a group have terminated and that one other work item of this group cannot: as a work item is not able to return from terminated to performing state, the system is blocked. In the context of our applications, abort is not acceptable and by hand recovery is necessary (but WorkCoordinator experts say that the case where a work item fails, is extremely rare).

\section{CORVETTE IMPLEMENTATION}

Corvette is a client for, on the first hand WorkCoordinator, and on the other hand Motu server. Concerning the interface between Corvette client and Motu server, as both are written in Java, Corvette client is simply a special Motu client. Concerning Corvette client and WorkCoordinator (written in $\mathrm{C}++$ ), the interface is based on WorkCoordinator IDL interface and on interoperability between Visigenic C++ $O R B$ and Visigenic Java ORB.

In order to detect work items creation and termination, as necessary for transaction management, triggers and Java stored procedures have been added to WorkCoordinator workflow relevant data managed by an Oracle server (Corvette trigger component). Each time a new work item is created or deleted, a message is sent to the Motu notifier component. Note that the installation of these triggers in the WorkCoordinator relevant data database is the only intervention done in some WorkCoordinator structures.

Finally, the Motu notifier is a special Motu server that monitors WorkCoordinator events and create a transaction each time a work item is started. It manages also notification information for users.

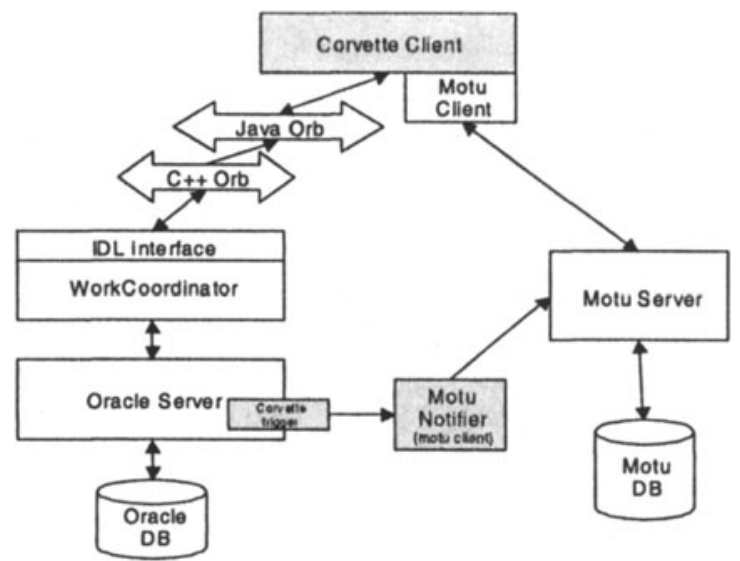

Figure 5 - Corvette implementation overview 


\section{SYNTHESIS AND LEARNED LESSONS}

Globally, this experiment is a success. We demonstrate the feasibility of defining a cooperative workflow system by "plugging" (in the sense of integrating software component without modification) together a "traditional" workflow system and an advanced cooperative transaction model. The main reason for this success is the absence of data flow consideration in WorkCoordinator. Thus, we did not have to manage the integration of WorkCoordinator data flow model with our transaction model. Another success is the demonstration of the ability to model cooperative processes as traditional processes, but to interpret them in a cooperative way corresponding to cooperative behaviours.

This success is limited in the sense that not all cooperation capabilities, initially forecasted, have been implemented in a so flexible way (due to inability to share intermediate results between succeeding activities). To surpass this limitation it is necessary to provide activities with the capability to anticipate: "anticipation is the weakening of strict sequential execution of activity sequences in a process by allowing intermediate results to be used as preliminary input of succeeding activities". For more about anticipation, see (Grigori, Charoy et al. 2001). Anticipation allows to implement Producer/consumer and Redactor/reviewer between succeeding activities, thus providing support for the full ECOO cooperation model.

As a conclusion of this experiment, we think that, if a workflow manager component does not impose constraints on dataflow, and if it provides the capabilities introduced in the previous paragraph (anticipation, events and group termination), it is possible to develop a cooperative workflow management system by simply plugging together this workflow component and a cooperative transaction manager. In addition, if all the activities of the process are concurrent, i.e. execute in isolation, as the process model does not change, this workflow management system has the behaviour of a traditional (competitive) one.

\section{REFERENCES}

1. Agostini, A. and G. De Michelis (1996). Modeling the Document Flow within a Cooperative Process as a Resource for Action, University of Milano.

2. BSCW "http://bscw.gmd.dell."

3. Canals, G., C. Godart, et al. (1998). A criterion to enforce correctness of indirectly cooperating applications. Information sciences.

4. Casati, F., Pozzi, G. (1999). Modeling Exceptional Behaviors in Commercial Workflow Management Systems. CoopIS.

5. CHI (1997). "Workshop on awareness in collaborative systems." http://www.usabilityfirst.com/groupware/awareness/workshop/.

6. CorvetteManual (2001). "http://lwww.loria.fr/equipes/ecoo/corvette/CorvetteUserManual.htm."

7. Dourish, P. (1998). "Using Metalevel Techniques in a Flexible Toolkit for CSCW Applications." ACM Transactions on Computer Human Interaction 5(2).

8. Ellis, C. and C. Maltzahn (1997). Chautaqua Workflow System. 30th Hawaii Int Conf. On System Sciences, Information System Track,.

9. Georgakopoulos, D. (1999). Collaboration Process Management for Advanced Applications. International Process Technology Workshop, Grenoble.

10. Godart, C. (1993). COO : a transaction model to support cooperating software developers. European Software Engineering Conference, Garmisch, Germany.

11. Grégori, N. (1999). Etude clinique d'une situation de conception de produit. Vers une pragmatique 
de la conception. Psychologie. Nancy, University of Nancy 2: 239.

12. Grigori, D., F. Charoy, et al. (2001). Anticipation to enhance flexibility of workflow execution. DEXA Conference, Munich, Lecture Notes in Computer Sciences.

13. Grigori, D., F. Charoy, et al. (2001). Flexible Data Management and Execution to Support Cooperative Workflow: the $\mathrm{COO}$ approach. The Third International Symposium on Cooperative Database Systems for Advanced Applications (CODAS'01), Beijing, China.

14. Han, Y., A. Sheth, et al. (1998). A taxonomy of Adaptive Workflow Management. Towards Adaptive Workflow Systems, CSCW'98 Workshop, Seattle, USA.

15. Heinl, P., S. Horn, et al. (1999). A Comprehensive Approach to Flexibility in Workflow Management Systems. Joint Conference on Work Activities Coordination and Collaboration (WACC'99), San Francisco.

16. Jablonski, S. and C. Bussler (1996). Workflow management - Modeling Concepts, Architecture and implementation, International Thomson Computer Press.

17. Joeris, G. (1999). Defining Flexible Workflow Execution Behaviors. Enterprise-wide and Crossenterprise Workflow Management - Concepts, Systems, Applications', GI Workshop Proceedings Informatik'99, Ulmer Informatik Berichte Nr. 99-07, University of Ulm.

18. Leymann, F. and D. Roller (1999). Production Workflow, Prentice Hall.

19. Motu (2001). http://motu. sourceforge.net.

20. Nutt, G. J. (1996). The Evolution Toward Flexible Workflow Systems. Distributed Systems Engineering.

21. Reichert, M. and P. Dadam (1998). "ADEPTflex - Supporting dynamic Changes of Workflows Without Losing Control." Journal of Intelligent Information Systems 10.

22. Reuter, A. a. S., F. (1995). "Contracts - A Low-Level Mechanism for Building General-Purpose Workflow Management Systems." IEEE Data Engineering Bulletin 18(1).

23. Skaf, H., F. Charoy, et al. (1999). "Maintaining Shared Workspaces Consistency during Software Development." Software Engineering and Knowledge Engineering 9(5).

24. Sourceforge "http://www.sourceforge.net."

25. Suchmann, L. A. (1987). Plans and Situated Action. The Problem of Human-Machine Communication. Cambridge University Press.

26. Teamscope "http://cscw.msu.edi/scope.html."

27. WCO (2001). http://www.hitachi.co.jp/Prod/comp/soft1/wco/eng/index.html,"

"Hitachi-WorkCoordinator,

28. Weske, M. (1996). Flexible Modeling and Execution of Workflow Activities. 31st Hawaii International Conference on System Sciences, Software Technology Track (Vol VII).

29. WfMC "Workflow Management Coalition." http://aiim.org/wfmc/mainframe.htm.

i Corvette stands for Coordination of a Virtual Team.

ii Corvette stands for Coordination of a Virtual Team. 